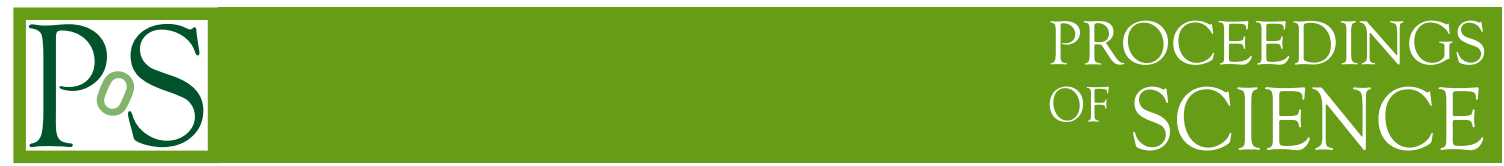

\title{
Electron - Ion Collider in China
}

\section{Xurong Chen*}

Institute of Modern Physics, Chinese Academy of Sciences, Lanzhou, 730000, China

E-mail: xchen@impcas.ac.cn

Electron Ion Colliders are regarded as a "super electron microscope" and can provide the clearest image inside the nucleon. It is the most ideal tool for us to understand the deep structure of the nuclear matter. In this contribution a polarized electron-ion collider plan in China (EicC) and its main physics goals will be introduced.

23rd International Spin Physics Symposium - SPIN2018 -

10-14 September, 2018

Ferrara, Italy

${ }^{*}$ Speaker. 


\section{Introduction}

The subatomic structure of the nucleon remains a frontier topic in hadronic physics research. One of the key goals in high- and medium-energy hadronic physics is to study the internal structure of the nucleon and to understand how QCD works in the non-perturbation region. For this topic, the primary experiment tool used is electron scattering from a nucleon or a light-nuclear target, from which the scattering cross section or spin-asymmetry can provide crucial information on the spin-averaged and spin-dependent parton distribution functions.

Electron Ion Colliders (EICs) [1] are regarded as a "super electron microscope" and can provide the clearest image inside the nucleon. It is the most ideal tool for us to understand the deep structure of the nuclear matter, especially the quark-gluon structure of the nucleon and nuclei. With high luminosity, broad kinematic range and various polarized ion beams, EIC will be superior to any facility of the past and present time. Polarized EICs are the next generation "multi-dimensional electron microscopes" that are most effective in studying the deep structure and strong interactions of particles. Currently there are several EIC plans over the world, such as electron Relativistic Heavy Ion Collider (eRHIC) [2], JLab Electron Ion Collider (JLEIC) [3] in USA, and LHeC [4] at CERN (unpolarized). The EIC plans have been discussed in many details in the last decade which are summarized in the EIC whitepaper [1] and the INT report [5].

\section{EicC Plan and Its Physics Goals}

Based on the HIAF (the Heavy Ion High Intensity Accelerator Facility, approved in 2015 in China), the Institute of Modern Physics, Chinese Academy of Sciences (IMP) is proposing to build a high luminosity polarized EIC facility in China, EicC, to carry out the frontier research on nucleon structure studies.

The EicC design parameters are summarized in Table 1.

\begin{tabular}{|c|c|c|c|c|}
\hline \hline Accelerator & Electron beam & Proton beam & $\sqrt{S}$ & Luminosity $\left(\mathrm{cm}^{-2} \mathrm{~s}^{-1}\right)$ \\
\hline EicC & 3.5 & 20 & 16.7 & $0.5 \sim 1.0 \times 10^{34}$ \\
\hline \hline
\end{tabular}

Table 1: EicC accelerator design parameters. Both electron and ion beams are polarized. Energy unit in $\mathrm{GeV}$.

We compare the EicC with the existing or planned ep facilities in Fig. 1. In Fig. 2 we show the kinematic ranges for the EicC and JLab $12 \mathrm{GeV}$ facility. Here we can see that the EicC is the best window for studying sea quarks. Unlike other EIC proposals [1, 2, 3, 4] where the focus is at high energy and small-x in order to probe the gluon's role to the inner structure of nucleons, EicC will focus on valance- and sea-quark contributions to nucleon structure and inter-nucleon effects.

\subsection{Study of nucleon structure}

EicC-I will allow a full study of the 1D and 3D structures of the nucleon. The 3D structures include transverse-momentum distribution (TMD) through measurements of the semi-inclusive deep-inelastic scattering (SIDIS) and Generalized Parton Distributions (GPD) through deep virtual Compton scattering (DVCS). High-precision measurements of the distribution functions of sea 


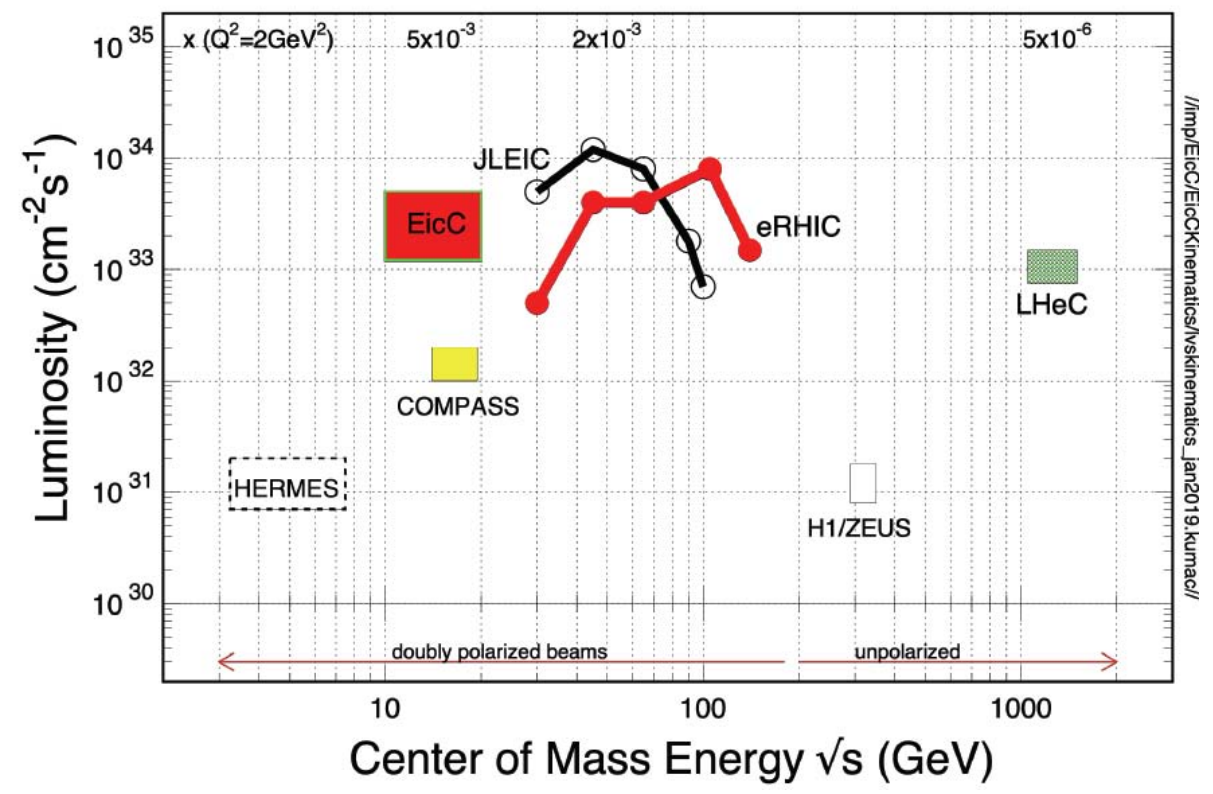

Figure 1: Compare the EicC with non-polarized and polarized electron-nucleon machines. The values of $x$ are also listed in the top of the figure.

quarks and valence quarks at EicC will uncover the internal structure of nucleons and ultimately solve the puzzles about nucleon properties.

\subsection{Explore proton mass QCD structure and Baryon States with Heavy Flavor}

Protons are made of three valence quarks. Yet the sum of masses of all quarks is far too small to explain the proton's total mass.

In $1995 \mathrm{Ji}$ [6] proposed the proton mass was decomposed into four parts: quark condensate, quark energy, glue field energy and trace anomaly. According to the current lattice QCD calculation [7], about $23 \%$ of proton mass comes from trace anomaly, but so far we know very little about its physics. Can JLab $12 \mathrm{GeV}$ and EIC machines get any insight on the proton mass QCD structure by decomposing it into the mass and motion of quarks and gluons? JLab 12 [8] will measure the $\mathrm{J} / \psi$ production near threshold (at small $\mathrm{Q}^{2}$ ). The center of mass energy $\sqrt{S}$ is about $15 \sim 20 \mathrm{GeV}$ for the EicC. This will provide a clean environment for studying the bottom-hadron, especially the $\Upsilon$. Compared to JLab $12 \mathrm{GeV}$, with higher center of mass energy, the EicC will offer precision measurement of $\Upsilon$ production near threshold. The heavier mass of the bottom should help to suppress the theoretical systematic uncertainties. This measurement will shed light on the proton mass study.

Another frontier that has attracted attention in the past year are exotic hadronic states, i.e., looking for hadronic states other than mesons and baryons [9, 10, 11, 12, 13]. Recently, two charmed pentaquark states have just been discovered at the LHC, triggering a research frontier to be formed to study the nature and properties of these exotic states in more detail. For Baryon States with bottom quark, EicC's advantages include: 1) larger cross section comparing to e+e- collision; 


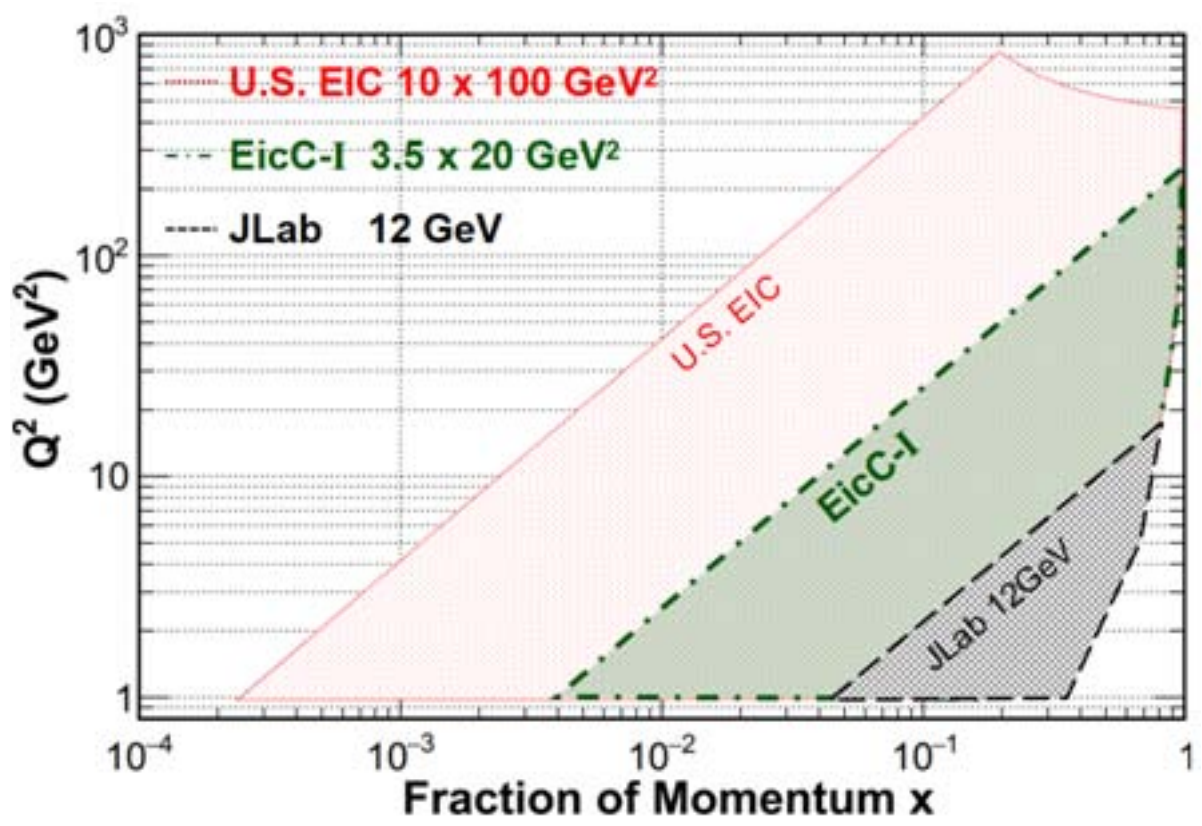

Figure 2: Compare the kinematic ranges of EicC with JLab $12 \mathrm{GeV}$, for DIS process.

2) smaller background comparing to pp and ppbar collision; and 3) Especially, the polarized EicC can well pin down the quantum numbers of the observed particles.

\subsection{Nuclear Medium Effects}

How does the nuclear environment affect the distribution of quarks and gluons and their interactions in nuclei? The third topic is to study medium effects include hadronization, EMC, SRC, color transparency, etc.

hadronization is a manifestation of the transition from quarks to hadrons. Due to the nonperturbative nature, it has been always challenge for QCD calculations. Since phenomenological models can not describe the whole set of experimental results, So new and more precise measurements are required. According to energy, there are two kinds of hadronizations: 1) at high energies the Hadron forms outside the medium; 2) at low energies: Hadron forms inside the medium. Due to its appropriate energy, the EicC is an ideal machine to study hadronizations both inside and outside the medium.

Of course, the EicC will offer more physics measurements than mentioned above. For example, the structure function measurements for both pions and Kaons.

\section{Conclusions}

EicC is complementary to JLab $12 \mathrm{GeV}$ and other EIC physics programs in U.S. and Europe. The highly polarized EicC plus the advanced high-tech detecting system will allow a series of world-class precision measurements for nucleon structure related physics. In addition to the advancement of the science, the EicC will also greatly boost the technology development. The EicC, 
with JLab $12 \mathrm{GeV}$, US and Europe ElC machines, will advance our understanding of gluons, sea and valence quark structures of the nucleon and the strong interaction.

The EicC machine will be constructed in Huizhou city, Guangdong province. We are pushing forward the EicC plan. Acceralator and detectors are under designing. Worldwide collaborations, include both theoretical and experimental, are highly needed.

Acknowledgments: We are grateful for many enlightening discussions with physics,detector and accelerator working groups. Especially thanks to J. P. Chen, N. Xu, and B. S. Zou for fruitful discussion.

\section{References}

[1] EIC-White Paper, Electron-Ion Collider: Next QCD Frontier,e-Print: arXiv:1212.1701 [nucl-ex].

[2] eRHIC Homepage, http://www.phenix.bnl.gov/WWW/publish/abhay/Home_ of _ EIC/.

[3] JLab EIC Homepage (https://eic.jlab.org/wiki/index.php/Main_Page).

[4] J. L. Abelleira Fernandez et al., A Large Hadron Electron Collider at CERN: Report on the Physics and Design Concepts for Machine and Detector, http://arxiv.org/abs/1206.2913.

[5] INT-Write-Up, Gluons and the quark sea at high energies: distributions, polarization, tomography, e-Print: arXiv:1108.1713 [nucl-th].

[6] X. Ji, Phys. Rev. Lett. 74, 1071 (1995); Phys. Rev. D 52, 271 (1995).

[7] Yi-Bo Yang, Jian Liang, Yu-Jiang Bi, Ying Chen, Terrence Draper, Keh-Fei Liu, and Zhaofeng Liu, Phys. Rev. Lett. 121, 212001 (2018)

[8] JLab 12 GeV Upgrade Homepage: http://www.jlab.org/12GeV/; J.P. Chen, H. Gao, T.K. Hemmick, Z.-E. Meziani, P.A. Souder, the SoLID Collaboration, A White Paper on SoLID (Solenoidal Large Intensity Device), : arXiv:1409.7741 (2014).

[9] Jia-Jun Wu, Lu Zhao, B. S. Zou, Physics Letters B 709 (2012) 70.

[10] Y. Huang, J. He, H. F. Zhang and X. R. Chen, J. Physics G 41, 115004 (2014).

[11] Y. Huang, J.-J. Xie, J. He, X. Chen and H.-F. Zhang, arXiv:1604.05969.

[12] S. Joosten, Z.-E. Meziani, PoS QCDEV2017 (2018) 017, arXiv:1802.02616v2 [hep-ex].

[13] S. Joosten, arXiv:1803.08615v1 [hep-ph]. 